\title{
Visages de l'idiotie dans Le Vent de Claude Simon
}

\section{Aude Michard}

\section{OpenEdition}

\section{Journals}

Édition électronique

URL : http://journals.openedition.org/ccs/633

DOI : $10.4000 /$ ccs.633

ISSN : 2558-782X

\section{Éditeur :}

Presses universitaires de Rennes, Association des lecteurs de Claude Simon

\section{Édition imprimée}

Date de publication : 31 décembre 2009

Pagination : 27-45

ISBN : 9782354120580

ISSN : 1774-9425

\section{Référence électronique}

Aude Michard, «Visages de l'idiotie dans Le Vent de Claude Simon », Cahiers Claude Simon [En ligne] 5 | 2009, mis en ligne le 21 septembre 2017, consulté le 01 mai 2019. URL : http:// journals.openedition.org/ccs/633; DOI : 10.4000/ccs.633 


\title{
Lectures du Vent
}

\section{Visages de l'idiotie dans Le Vent de Claude Simon}

\author{
Aude MICHARD
}

Au monde il y a les crédns, les imbéciles, les stupides et les fous. [...] la personne normale est celle qui mêle en une mesure raisonnable toutes ces composantes, ces types idéaux ${ }^{2}$.

Le personnage du notaire dans Le Vent. Tentative de restitution d'un retable baroque de Claude Simon approuverait sans aucun doute cette proposition tirée du Pendule de Foucault d'Umberto Ecco. Il ajouterait que la "personne normale », qu'elle appartienne davantage à la catégorie des crétins, des imbéciles, des stupides ou des fous, se reconnaît d'abord à ses motivations intéressées. Un seul individu dans le roman de Claude Simon échappe à cette tyrannie de l'intérêt, c'est l'idiot Antoine Montès :

Un idiot. Voilà tout. Et rien d'autre. Et tout ce qu'on a pu raconter ou inventer, ou essayer de déduire ou d'expliquer, ça ne fait encore que confirmer ce que n'importe qui pouvait voir du premier coup d'œil. Rien qu'un simple idiot. Seulement, lui, avec le droit de se promener en liberté, de parler aux gens, de signer des actes et de déclencher des catastrophes. ( $V$, incipit)

\footnotetext{
${ }^{1}$ A soutenu en 2009 une thèse de doctorat sur La représentation des lieux dans les romans de Claude Simon (voir rubrique "Actualité de l'œuvre»).

${ }^{2}$ Umberto Ecco, Le Pendule de Foucault, Paris, Le Livre de Poche, 1990, traduction de JeanNoël Schifano, chapitre X, p. 65.
} 
En la magistrale ouverture du Vent tout le roman est contenu. Le récit témoignant des aventures de l'idiot est un récit à la croisée du roman de formation et du roman de (més)aventures. Le respect de la chronologie des événements, à l'exception d'une importante analepse entre les chapitres XI et XIII, l'organisation en chapitres, de même que certains personnages qui constituent encore des « vestiges balzaciens ", tels que l'oncle ou Maurice, témoignent d'un respect relatif des conventions du roman traditionnel dans Le Vent. Cependant, selon le titre de l'article de Claude Sarraute consacré à La Route des Flandres, nous pouvons dire que "Simon affirme sa manière $"^{3}$ dès Le Vent par le truchement de la "vulgaire et idiote aventure d'un vulgaire idiot $»$. L'irréfragable beauté de ce roman et le charisme de son étrange héros nous conduisent à nous réinterroger aujourd'hui sur ces visages ou figures de l'idiotie qui ornent le roman-retable, et à redire dans quelle mesure le regard singulier que pose l'idiot sur le monde peut servir d'introduction à une théorie d'écriture, à un art poétique. Nous nous attarderons donc sur la généalogie de l'idiot simonien, c'est-à-dire sur ses ancêtres en littérature, puis sur les descendants de Montès dans le roman simonien, ce qui permettra, peut-être, de mettre en exergue ce qu'a d'idiote l'écriture simonienne elle-même.

Dans Le Vent aucun personnage n'est à proprement parler déficient intellectuellement, ou atteint d'une maladie telle que l'épilepsie du prince Muichkine chez Dostoïevski ${ }^{4}$. Pourtant, si l'on tient compte de la trentaine d'occurrences du mot " idiot " (substantifou adjectif) et de la démultiplication de ses synonymes ("imbécile», " crétin » et " cloche »), aucun personnage du roman n'est épargné. Nous pouvons déjà dire que ces termes seront repris dans toute leur variété dans les romans suivants, notamment avec le "pauvre con" dans La Route des Flandres et le "vieux con" dans Histoire. L'idiot étant communément celui qui parle, raisonne ou agit d'une façon irrationnelle pour un autre, c'est la question de la communication, de l'altérité ${ }^{5}$ qui est induite ici, comme dans bien des romans de Simon.

\footnotetext{
${ }^{3}$ Le Monde, 8 octobre 1960.

${ }^{4}$ Fédor Dostoïevski, L'Idiot, (1868), Paris, Le Livre de Poche, trad. G. Arout, 1972.

${ }^{5}$ Étymologiquement, idiotes désigne l'unique, le particulier, avant de devenir le commun puis l'ignorant.
} 
La crise de la communication postulant une crise du sens, chacun est potentiellement l'idiot d'un autre. À l'exception de Montès, qui est considéré à l'unanimité comme l'idiot par excellence, nous allons voir que s'il $\mathrm{y}$ a un idiot il $\mathrm{y}$ en a très souvent au moins un second. On trouve en ville deux grandes catégories d'idiots : ceux qui ont une appréhension subjective et sensible du monde, tels que Cécile et Montés, et ceux qui appréhendent le monde d'une manière rationnelle et logique, tels que le notaire, Hélène ou le narrateur. Confrontés à la première catégorie, ceux de la seconde sont à leur tour frappés d'idiotie, comme par exemple le notaire face à Montès : " [...] il en était arrivé à se demander lequel des deux, de lui ou de l'autre, était l'idiot, ou le fou $[\ldots] »(V, 14)$. On trouve ainsi plusieurs couples d'idiots par contamination : au chapitre VI, lors de leur " étrange et nocturne duo d'amour », Rose et Montès se retrouvent, dit Rose, « comme deux cloches sur ce banc» (74); au chapitre XVI c'est Cécile et son fiancé à l'hôtel qui seront à leur tour " plantés là comme deux idiots" (178).

Cécile est désignée par son père dialoguant avec Hélène comme " ton idiote de sœur" ( $V, 130)$, et s'était du reste qualifiée ainsi dans son billet à Montès : " Je me suis conduite comme une idiote " (123). Cette même Cécile houspillera son fiancé : "Cessez donc de faire l'idiot à la fin " (178), ou encore " fichez-moi le camp, espèce d'idiot» (180). Quant à l'oncle de Montès, il est dans la bouche de Cécile " mon imbécile de père », et dans celle de Maurice "votre imbécile de père " (132). Montès lui-même confessera son idiotie après sa visite au quartier gitan : "J'ai été idiot " (98). Dans la situation spatio-temporelle et sociale du roman, c'est-à-dire celle d'une ville de taille moyenne dans les années 50, ce qui caractérise les idiots est d'abord leur inobservance des lois et des conventions sociales. C'est particulièrement le cas de Montès, agissant «toujours avec ce même mépris, ou refus, ou ignorance des conventions, des convenances " (81), ou de Cécile, qui à la stupeur générale a « couru après Montès avec ce mépris non seulement de toute pudeur, de toute convenance, mais encore de toute habileté (ce qui est peut-être en somme la même chose) " (172). Mais c'est surtout l'absence de motivation intéressée, ou plus exactement le mépris qu'ils ont de leur propre intérêt, qui scandalise si fort la «ville». 
L'idiot est donc d'abord seul face au groupe, il s'en distingue par son attitude ou sa présentation excentrique : il y a « l'idiot du village " comme celui de la famille, et lorsque l'écart d'avec la norme est par trop conséquent, son attitude est considérée comme de la démesure, qui est dès le Moyen Âge liée à la folie. La tenue vestimentaire de Montès est décalée parce que démodée, hors de saison. Par son indifférence manifeste à l'égard du bon goût ou de la fonction sociale du vêtement, l'idiot est remarquable par sa tenue insolite : Montès traîne quelle que soit la saison le même imperméable usé " qu'aucun clochard de la ville n'aurait seulement voulu » $(V, 7)$, et inflige à la vue de tous "sa bizarre dégaine - de défroqué, dirent les uns, d'échappé d'asile, dirent les autres " (11-12). L'idiot est inquiétant parce qu'il a l'air fou : comme Perceval chez Chrétien de Troyes, qui portait l'habit du "Gallois » confectionné par sa mère, Montés semble n'avoir porté toute sa vie que l'uniforme choisi par la sienne :

[...] une sorte d'uniforme pour ainsi dire, qu'il portait sans doute depuis sa quinzième ou seizième année sans avoir eu l'idée d'en changer parce que la femme (sa mère) qui les lui avait choisis une première fois n'avait sans doute elle-même jamais eu l'idée qu'il pût s'habiller d'une façon qui [...] différât sensiblement de la tenue d'un boy-scout. ( $V, 55-56)$

L'idée du costume de cirque est ensuite introduite par Maurice qui compare Montès à Grock (99). Plus bas, Cécile, le voyant aux prises avec la veste qui refuse de s'enfiler, s'impatiente : "C'est un numéro de cirque ? " (117), puis l'accuse de jouer la comédie, " de [se] rendre ridicule à plaisir, de [s'] habiller comme un clown » (118). Plus loin le narrateur évoque de nouveau " ses vêtements râpés, son bizarre visage de clown ou de traître de comédie " (159). Lorsqu'il se trouve empêtré dans les vignes du domaine paternel en compagnie du régisseur : « [...] quelqu'un d'étranger qui se fût trouvé là n'eût pu se retenir de rire à ce spectacle caricatural, bouffon [...]»(20).

Montès provoque la stupeur, l'agacement, la colère, mais également le rire, un rire iconoclaste sans aucun doute, puisque nous dirons également la dimension christique de ce héros idolâtre. Rappelons que la distraction perpétuelle et méditative de Montès, sa lenteur affective et sensible, causent son retard par rapport aux événements 
dont il ne prend conscience qu'après-coup. Sa position de décalage le déséquilibre, il va à contretemps, il titube comme l'ivrogne baroque. Tout ce qui cloche chez Montès (Rose le qualifie d'« espèce de cloche dans un hôtel pour cloches ») s'accompagne d'une expression orale souvent contrariée : il " bégayait de plus belle " $(V, 78)$, " bredouille, bégaye " et produit des " sons inarticulés " (101) en " bégayant, s'embrouillant» (158). Il exaspère ainsi Rose une nouvelle fois : "Qu'est-ce que vous dites ? Vous ne pourriez pas arrêter de bafouiller? » (58).

Nous souhaitons relever à présent quelques caractéristiques du profil de l'idiot simonien qui marquent une lourde hérédité littéraire. Le sens du mot " idiot » entre les XII" et XVII ${ }^{\circ}$ siècles coïncide avec celui d'ignorant : le naïf est toujours en situation d'apprentissage. De fait, Le Vent est à bien des égards un roman d'apprentissage dans la lignée du roman de chevalerie puis du roman picaresque, jusqu'au roman réaliste et existentialiste. Quelques visages d'idiots nous viennent à l'esprit à la lecture du Vent : Perceval le Gallois, Don Quichotte, Candide, Félicité des Trois contes de Flaubert, François le Champi, le Prince Muichkine, Benjy Compson et Joe Christmas chez Faulkner, et même Roquentin ou Meursault.

Ignorant, l'idiot s'apparente à un animal, une bête rétive apparemment à toute forme de domestication : Montès est comparé maintes fois à un chien (battu) et à un singe, Cécile à un chat ou un oiseau. Cécile rend visite à Montès de même « que s'il eût été un animal rare ou inconnu dans un zoo, une curiosité " $\{V, 46)$. Comme l'animal, il est silencieux ou produit, nous l'avons dit, des "sons inarticulés »: Montès à ce titre rappelle le « ravi » ou " champi » de George Sand, ou Flaubert lui-même selon l'étude sartrienne L'Idiot de la famillé ${ }^{6}$ : son mutisme et sa retenue lui donnent un aspect énigmatique et vaguement inquiétant qui l'apparentent à la brute ou à l'aliéné.

${ }^{6}$ Jean-Paul Sartre, L'Idiot de la famille. Gustave Flaubert de 1821 à 1857, Paris, Gallimard, 1971. 
Innocent, le visage de l'idiot coïncide aussi avec celui de l'enfant : les visages innocents des enfants sont, rappelons-le, le sujet de prédilection des photographies de Montès ${ }^{7}$. L'idiot ressemble d'ailleurs physiquement à un enfant : Montés aurait vieilli pendant l'enfance selon le narrateur, et comme pour Muichkine chez Dostoïevski ou Benjy chez Faulkner, on ne sait trop quel âge lui donner. Montés se tient "à la façon des écoliers, les deux bras en corbeille derrière son dos» $(V, 9)$, exhibe un " buste maigre, dépourvu de poils » (101), et face au policier (chapitre XIII) il a " l'élémentaire réflexe physique du gosse qui lève le coude dans la crainte de recevoir une autre beigne " (139).

L'idiot, qui est un héros en formation, est par conséquent aussi un héros errant, un déraciné. Montés comme Muichkine retourne dans la ville où il a été conçu mais qu'il n'a jamais connue ; quoique pour ce qui concerne Montés il soit vraisemblablement étranger à ce monde même : " toujours insensible semblait-il au monde qui l'entourait, le visage toujours absent $"(V, 81)$. Sans domicile fixe, il élit des lieux de passage, anonymes : la route, la place, le carrefour, l'hôtel. Montés et Muichkine se retrouvent sur un banc lorsqu'ils ne sont pas dans leur chambre d'hôtel, Antoine Roquentin chez Sartre ${ }^{8}$, qui fait lui aussi partie des ancêtres en littérature d'Antoine Montés, passe le plus clair de son temps entre le café et le jardin public. La progression de Montés sur les routes prend l'allure d'une lutte contre une force cosmique, que symbolise dans Le Vent la tramontane, et qui est la masse du temps. Le Rossinante de Don Quichotte est pour Montés remplacé par une bicyclette, l'épée du chevalier par l'appareil de photographie, et les moulins à vent par le vent lui-même. La conscience aiguë de l'écoulement du temps donne le vertige à l'idiot qui voudrait mettre fin à cette giration incontrôlable, à cet « [...] éternel recommencement, l'éternel cheminement de la matière inerte, insensible, tournant dans l'infini, se déplaçant avec cette foudroyante et implacable lenteur, promesse d'un lent supplice, d'une lente agonie $[\ldots] »(\boldsymbol{V}, 56)$. L'oxymorique « foudroyante lenteur " est la cause du vertige épileptique de Montés (pour les idiots Muichkine et Flaubert le vertige est littéral). La marche par consé-

\footnotetext{
${ }^{7}$ « [...] purs, sinon des barbares et primitifs instincts - cruauté, violence, possession - du moins de leur conscience, et par conséquent du mal $[\ldots] »(V, 115)$.

${ }^{8}$ La Nausée, Paris, Gallimard, "Folio », 1938.
} 
quent, horizontale, équilibrante, s'apparente alors à un effort pour contrebalancer le mouvement de chute violent et imprévisible qui saisit l'idiot lorsqu'il prend conscience de l'écoulement inexorable du temps. Malheureusement l'errance restera sans terme comme l'écoulement du temps : Montès et Muichkine à la fin du roman n'ont vécu qu'une révolution, une progression égale à zéro. Muichkine retourne à son état d'ahurissement infantile, Montés quant à lui restera figé dans un " au-delà du désespoir », condamné à demeurer toujours " absent (ailleurs, ou plutôt nulle part [...]) » (158), ou encore « prisonnier de la marge », selon l'expression de Michel Foucault au sujet de l'aliéné 9 .

Le premier personnage simonien, Louis dans Le Tricheur, était déjà un marginal, errant sur les routes et d'hôtel en hôtel à la recherche de petites «combines idiotes" (Tr.,183). Tous les grands personnages simoniens sont des marginaux, et avec Montés, qui est le plus marginal de tous, Simon rend un hommage explicite à Dostö̈evski.

Lorsque le roman de Simon s'ouvre in medias res sur ces mots du notaire : "Un idiot », il se propose délibérément comme relecture du roman intitulé L'Idiot, de même qu'Aimé Césaire avec Une Tempête relisait La Tempête de Shakespeare. Nous laisserons de côté les influences de Dostoïevski sur la poétique du roman et nous consacrerons exclusivement ici à relever les similitudes entre les deux visages d'idiots.

Soulignons d'abord quelques éléments d'intertextualité dans la trame du roman. Les deux héros idiots font un voyage en train - en troisième classe - et tous deux pour se rendre dans un lieu inconnu, où personne ne les attend, afin de toucher un héritage, car ils sont les derniers porteurs du nom de la famille. C'est le même personnage du « cousin éloigné et pauvre, et même déclassé, que l'on n'a jamais vu en raison d'une de ces histoires de famille » $(V, 44)$. Ils louent tous deux une chambre sombre qui donne sur une cour déserte. L'entourage de l'idiot est constitué de personnages hétéroclites issus 
de toutes les classes sociales. Dans Le Vent, Jep et son épaisse chevelure noire est le descendant de l'ombrageux Rogojine, Maurice est l'équivalent des petits malfaiteurs Hippolyte et Terentiev qui tentent de faire chanter le prince, il est également assoiffé de considération comme Gania. On trouve dans les deux romans les deux figures féminines des amoureuses : la jeune cousine de bonne famille (Aglaë et Cécile) et la putain (Nastassia Philippovna et Rose) ${ }^{10}$. Tous entretiennent avec l'idiot une relation ambiguë d'affection et de haine ; l'idiot indigne et fascine à la fois, et l'on s'interroge sur ses motivations profondes : « [...] un type comme ça, dit le notaire, c'est qu'il est, ou bien très malin, ou un parfait idiot [...] » (17), «Ce prince, dit Alexandra Epantchine, est peut-être un malin et nullement un idiot $»^{11}$. L'histoire de Muichkine n'est rien de plus qu'« une histoire idiote qui tient en deux mots $\|^{12}$ comme celle qu'évoque le général Ardalianovitch, et rappelle le jugement du narrateur sur celle de Montès, qui ne serait que " la vulgaire et idiote aventure d'un vulgaire idiot» (82).

À l'instar du fou médiéval ou du gracioso du théâtre baroque, l'idiot possède une aptitude prémonitoire, et son sentiment de la fatalité apparaît dès lors qu'il se sent impliqué de manière affective :

[...] [Muichkine] avait grande envie de laisser tout cela et de retourner d'où il était venu, d'aller dans un endroit perdu [...]. Il pressentait que s'il restait ici [...] il serait pris sans retour par cette existence [...]. Mais il ne mit pas dix minutes à conclure qu'il lui était impossible de fuir ${ }^{13}$.

\section{Quant à Montès :}

[...] sa première impulsion donc, ayant été de s'en aller, de monter faire ses bagages et quitter l'hôtel le soir même. $(V, 56)$

[...] mais [...] l'endroit où en réalité il voulait être, ne pouvait qu'être, c'était là où il se trouvait. (60)

\footnotetext{
${ }^{10}$ La violence autodestructrice de la " femme outragée " chez Nastassia apparaît aussi chez Cécile et la mère de Montès.

${ }^{11}$ L'Idiot, op. cit, p. 79.

${ }^{12}$ Ibid, p. 159.

${ }^{13}$ Ibid., p. 451.
} 
Alors qu'il n'aspire qu'à la paix, les tensions autour de l'idiot deviennent palpables et les incidents éclatent. Sa confrontation au monde " confine à l'anecdote $»^{14}$, tant ses espérances sont systématiquement et mécaniquement pulvérisées, à l'image de celles de Candide, l'optimiste " boy-scout» de Voltaire, qui découvrait le monde par des mortifications successives - un monde qui ne pouvait être pire. Le caractère ambigu de l'idiot réside dans l'écart entre son aspiration au calme et les déboires que ses actions entraînent ${ }^{15}$. L'épisode repris fidèlement par Simon est celui de l'assassinat de la femme aimée par son propre amant, et d'un coup de couteau dans la poitrine : Nastassia poignardée par Rogojine et Rose par Jep. Les deux idiots ont du reste le sentiment de leur nocivité : Muichkine pense qu'« un démon étrange et effrayant s'était accroché à lui définitivement et ne voulait plus le quitter $»^{16}$, et Montès pressent « un danger accroché à lui, inséparable de lui, comme un chancre, un cancer» $(V, 116)$. L'aliénation du personnage est lisible à maintes reprises déchiré entre la nécessité de s'arracher à la sensation qui rend trop vulnérable, et son tempérament naturellement passif, Montès subit l'affrontement en lui de deux instances, il a « [...] cette sensation de promener comme une sorte de double, ou plutôt [...] une carcasse encombrante, abusive» $(V, 161)$.

L'aliénation, c'est aussi celle du narrateur, autre visage de l'idiotie du roman simonien. Lorsqu'au début du roman le notaire lui parle de Montès en ces termes : « [...] vous vous apercevez tout à coup que depuis un bon moment il ne vous écoute plus [...] », le narrateur ajoute : « Mais je ne l'écoutais plus [...]» (14). Un peu plus bas le «Pauvre type! " lancé par le notaire pourrait aussi bien être une réponse au narrateur lui-même qu'une insulte adressée à Montés absent. Enfin le narrateur se trouve en contact avec le notaire au sujet d'une « bicoque, une ancienne bergerie dans la montagne [qu'il s'est] mis en tête d'acheter et d'aménager » (83), comme Montés s'est mis en tête de reprendre la vieille baraque paternelle. Immergé dans l'histoire de Montés, le narrateur s'identifie à lui en vue de restituer les chaînons manquants : « Et au fur et à mesure qu'il me racontait

\footnotetext{
${ }^{14}$ Ibid., p. 56.

${ }^{15}$ Voir Jean Duffy, «Antithesis in Simon's Le Vent : authorial red herrings versus readerly strategies ", Modern Langage Review, 83 (3), juillet 1988.

${ }^{16}$ L'Idiot, p. 341.
} 
la scène il me semblait maintenant la vivre mieux que lui-même, ou du moins pouvoir en reconstituer un schéma sinon conforme à ce qu'il avait réellement été, en tout cas à notre incorrigible besoin de raison $[\ldots] »(108)$. Le narrateur est un idiot puisqu'il s'identifie à un idiot et parce qu'il s'attaque à une entreprise idiote : la tentative de restitution d'une aventure passée - idiote de surcroît - à laquelle il n'a pas personnellement participé et dont il dénonce dès les premières pages du roman la vanité ${ }^{17}$. Il y a donc deux types d'idiotie : celle de la perception du réel ${ }^{18}$, et une sorte $\mathrm{d}^{\prime}$ « idiotie au second degré ", lorsque ayant pris acte de l'irréductible idiotie de toute perception, le narrateur (comme l'écrivain) s'acharne à convertir cette perception subjective, incohérente, incommunicable, en données lisibles et organisées d'après le système conventionnel de la langue.

Pour en revenir à l'aliénation du prince Muichkine, Hippolyte finit par dénoncer sa responsabilité dans les catastrophes : "C'est vous, vous, âme mesquine et mielleuse de jésuite, idiot, millionnaire-bienfaiteur [...], c'est vous qui avez suscité tout cela! $»^{19}$. Chez Simon, c'est le narrateur qui finira par éclater : "Bon Dieu! Mais qu'est-ce que vous attendez pour rentrer dans un couvent ? [...] Oui. Figurez-vous. Ç'a été inventé exprès pour ça. Pour protéger les types dans votre genre, pour les mettre hors d'état de nuire " $(V, 186)$. Cette suggestion d'aller s'enfermer au couvent est en adéquation avec le caractère monacal et ascétique du personnage.

La dimension christique du personnage de l'idiot est fondamentale. Le général Epantchine dit du prince : «C'est Dieu qui me l'en-

${ }^{17}$ Le narrateur s'efforçant à la reconstitution de l'histoire de Montès a recours au ciment de la logique et des déductions pour satisfaire son besoin de raison. Cependant les lacunes dans le récit que Montés fait au narrateur causent des irrégularités dans le texte. Par exemple, lorsque Montés se rend dans le quartier gitan avec Thérésa, le narrateur se fait la réflexion qu'« [i]l ne [lui] dit pas non plus s'il lui acheta des bonbons » $(V, 92)$. Pourtant, plus loin dans le récit, la fillette l'attend " tenant encore dans une de ses mains le sac de bonbons " (96). Il est également question au chapitre XI des « cinq ou six apéritifs » qu'a pris Maurice et qui deviennent au chapitre XIII " trois ou quatre pernods» (126 et 134). Enfin Thérésa peut avoir « huit ou dix ans » à l'arrivée de Montés et quelques mois plus tard l'assistante sociale lui apprend qu'elle a "déjà presque 13 ans " (33 et 160).

${ }^{18}$ Voir Clément Rosset, Le Réel. Traité de l'idiotie, Paris, Minuit, 1977, p. 40 et suivantes " [...] tout ce qui arrive est, de toute façon, également "idiot". [...] stupide, sans raison, comme l'est l'infinité des possibles, mais aussi simple, unique, comme l'est la totalité du réel ».

${ }^{19}$ L'Idiot, p. 439. 
voie », et plus bas son épouse : «je crois que c'est pour moi que Dieu vous a envoyé de Suisse à Pétersbourg $»^{20}$. Après l'avoir pris pour un simple d'esprit le notaire envisage que Montès sorte d'une "Trappe quelconque $^{21} »(V, 7)$. Lorsqu'il joue avec les filles de Rose, on peut lire "sur son visage une expression radieuse, ravie " (40), qui nous rappelle que le « ravi », variété provençale de l'idiot, est celui qui est proche de Dieu. Les moments d'hébétude de l'idiot sont des moments de contemplation, Flaubert dirait d'« extase». Notons encore que l'histoire de Montès s'apparente à celle de la pièce de Calderón La Dévotion à la croix évoquée dans le roman.

La vision « boyscoutesque » du monde de Montés, et cette façon qu'il a de "détourner charitablement la tête " face à un Maurice "pathétique et suppliant comme s'il quêtait, quémandait quelque chose " $(V, 105)$, le condamnent à être investi du rôle de confesseur. Rose lui " demande pardon " pour s'être emportée contre lui (70), Cécile lui écrira un mot d'excuse pour le même motif, enfin Maurice, après avoir tenté de le faire chanter avec le billet de Cécile, viendra lui-même faire sa confession au chapitre XI. À la stupeur de Montés, en moins de deux jours deux femmes (Rose et Cécile) lui posent la même question : "Pour quoi vous prenez-vous ? Pour un saint ?» (78 et 119). Le prince Stsh... dans le roman de Dostoïevski avait quant à lui bien discerné chez Muichkine le désir « de réaliser le paradis sur terre $»^{22}$

Deux descriptions dans Le Vent font coïncider le visage de Montés et celui du Christ : la description de la ville au chapitre III et celle de l'église au chapitre XVI. Montés a une physionomie d'homme coupable ${ }^{23}$, de ceux « qui, même victimes, passifs, portent dans une certaine mesure le poids d'une sorte de malédiction et de culpabilité » $(V, 168)$. Martyrisé, l'idiot subit non seulement la mortification morale de la mauvaise tournure des événements, mais encore des agressions physiques telles que les gifles (données par Gania à Muichkine ou par le policier à Montés), le coup de poing asséné par le gitan Jep, ou le coup de couteau de Rogojine. Le motif de la passion christique est récurrent dans Le Vent, comme lors de l'ascen-

\footnotetext{
${ }^{20}$ Ibid, p. 72 et 119.

${ }^{21}$ La mélancolie de Montès a du reste quelque chose de l'acédie.

${ }^{22}$ L'Idiot, p. 498.

23 " avec ce regard coupable, charbonneux" $(V, 159)$.
} 
sion par Montès de l'escalier de l'hôtel, après qu'il a tenté de digérer le coup de poing : Maurice observe du haut de l'escalier « l'ombre difforme [qui] poursuivait son ascension solitaire avec une sorte de pathétique et lent acharnement, d'obstination têtue, tragique " (99). Muichkine voit dans Rogojine son Judas ( $\mathrm{j}^{\mathrm{j}}$ attendrai, dit-il, avant de juger ce Judas »), et Montès aurait pu dire « il va me vendre » plutôt qu'« il va me le vendre » au moment fatal où Maurice s'apprête à lui révéler le vol de Jep. Stuart Sykes avait déjà proposé de lire le « Fichez-moi tout de suite le camp " de Montés à Maurice comme un "Arrière Satan ${ }^{24}$ qui confirme l'idée de reprise à " une thématique quasi religieuse empruntée au retable ", illustrant ainsi le sous-titre du roman «Tentative de restitution d'un retable baroque».

Montés, à la différence de Muichkine, est athée. Il a cependant à l'esprit le tableau d'une crucifixion aperçu au foyer où il visite les fillettes :«[...] un grand tableau sombre, à l'huile [...] sur sa gauche, vaguement : quelque chose avec des femmes debout dans des voiles bleus, et des clous, et des gouttes de sang habilement peintes sur les pieds percés, et un ciel noir $[\ldots] »(V, 183)$. Quoique la description n'ait rien de "vague », Montés qui analyse les détails refuse de comprendre l'ensemble, contrairement au prince qui, frappé par la violence du tableau de Holbein au-dessus de la porte chez Rogojine, y voit la transposition de sa propre histoire :

La nature apparait, en regardant ce tableau, sous la forme d'une bête énorme, implacable et muette [...] sous l'aspect de quelque immense mécanique d'une construction des plus moderne qui aurait absurdement happé, broyé et absorbé, sourde et insensible, un Être dont la grandeur est sans prix ${ }^{25}$.

"Implacable et muette ", c'est aussi la tramontane dans le roman de Simon, l'« immense mécanique [...] absurd[e] », c'est aussi le temps chez Simon comme chez Dostoïevski. Cette mécanique du temps est également l'une des forces œuvrant à la destruction des héros du romancier américain William Faulkner, auquel Le Vent rend également hommage.

\footnotetext{
${ }^{24}$ Stuart Sykes, "Le Vent ou le simulacre de l'ordre », Les Romans de Claude Simon, Paris, Minuit, 1979, p. 30. Voir aussi Jean Duffy, "Antithesis in Simons Le Vent», art. cit.

${ }^{25}$ L'Idiot, p. 597.
} 
On sait l'admiration de Simon pour Faulkner, dont il dit au cours de son entretien avec André Bourrin : «Ce que je préfère en Faulkner c'est son côté joycien ou proustien. [...] Faulkner c'est le Picasso de la littérature ${ }^{26}$. Dans le roman de Faulkner Le Bruit et la Fureur, Quentin, le fils aîné des Compson, se sent broyé par l'écoulement du temps : «Le Christ n'a pas été crucifié : il a été rongé par le menu tic-tac de petites roues $\|^{27}$; Benjy, l'idiot furieux, fait lui aussi l'orgueil de sa mère, et comme Montès stigmatise la violence et la folie familiales. L'idiot est le fruit d'une faute : Benjy est le fruit de l'amour incestueux de Mme Compson avec son frère Maury ${ }^{28}$, quand Montès est l'instrument de la vengeance maternelle en subissant avant sa naissance un arrachement haineux à sa terre d'origine.

Le monologue intérieur du sourd et muet Benjy, qui constitue la première partie du roman de Faulkner, témoigne d'une perception du monde exclusivement sensible, à l'instar de celle de Montès et de bien d'autres personnages simoniens : « je pouvais sentir» dit-il, «je pouvais entendre », « je pouvais aussi voir », autant de futurs leitmotive simoniens. Un élément caractéristique de la perception du héros autiste est l'apparente indépendance des objets :

$[\ldots]$ le sol montait toujours $[\ldots]$

[...] les marches de la cave ont grimpé la colline dans le clair de lune $[\ldots]^{29}$

Pour Montès, c'est « le plafond [qui] bascul[e] tout à coup à la verticale " $(V, 102)$. À cette perception altérée du réel correspond l'expression désordonnée de l'idiot, dont nous avons dit pour Montès qu'elle était essentiellement bégaiement et bafouillages. L'aven-

\footnotetext{
${ }^{26}$ Entretien avec André Bourrin, Les Nouvelles littéraires, 22 décembre 1960. Voir aussi " [...] maintenant plus personne ne peut être peintre sans ridicule à cause de Picasso. " $\{\mathrm{La}$ Corde raide, p. 177).

${ }^{27}$ Le Bruit et la Fureur [1929], Paris, Gallimard, «Folio », 1972, traduction Maurice-Edgar Coindreau, p. 100.

${ }^{28}$ Joe Christmas dans Lumière d'Août, descendant des amours d'un homme noir et d'une femme blanche, est également condamné à l'errance. Notons l'âge de ces héros christiques trente-trois ans pour Benjy et Christmas, "moins de trente-cinq " pour Montès.

${ }^{29}$ Le Bruit et la Fureur, p. 39 et 59.
} 
ture de l'idiot est d'abord une histoire dite par un idiot, " a tale told by an idiot ». Ces derniers mots tirés de la scène 5 de l'acte $\mathrm{V}$ de Macbeth de Shakespeare précèdent immédiatement ceux qu'emprunte Faulkner pour le titre de son roman : " full of sound and fury ». La confusion des dialogues et des descriptions, la déconstruction de la chronologie et la progression par associations analogiques dans le récit de Benjy, ont pour équivalent le récit fragmenté et incohérent de Montès aphasique au narrateur.

Pour ce qui concerne " le bruit et la fureur" dans Le Vent, avec les " bruits de la ville » et la polyphonie des instances narratives, depuis le salon de coiffure jusqu'à la terrasse de café, où Montès luimême raconte son histoire au narrateur, en passant par le bureau du notaire, chacun rapporte sa propre version ou tentative de restitution de l'histoire. Toutes les voix se font entendre à la fois, parlent à tort et à travers, se chevauchent, se contredisent, à l'image des tirades vides de sens de Maurice. Le brouhaha général confine au silence et celui qui s'exprime dans l'excès ne dit rien sinon son incapacité à s'exprimer ${ }^{30}$. Un bruit de fond couvre le brouhaha idiot de la ville : celui du vent, vide aussi de toute signification. Il est la manifestation de la fureur à l'échelle cosmique, une force élémentaire qui se débonde sans fin et qui, contrairement à la furibarde Cécile, est indestructible. Générateur de désordre, comme Montès, le vent est l'autre grand visage de l'idiotie dans le roman. D'ailleurs l'explicit du Vent rappelle curieusement celui du roman de Faulkner, lorsqu'après une crise de Benjy qui a vu la voiture prendre un autre itinéraire, tout rentre dans l'ordre :

La fleur brisée pendait au poing de Ben, et ses yeux avaient repris leur regard bleu, vide et serein, tandis que, de nouveau, corniches et façades défilaient doucement de gauche à droite ; poteaux et arbres, fenêtres et portes, réclames, tout dans l'ordre accoutumé ${ }^{31}$.

Dans Le Vent, après une longue description des rues immuables de la ville, le narrateur conclut sur ces mots : « [...] tout de nouveau dans l'ordre reformé, indestructible, jusqu'au vent lui-même, de nouveau là $[\ldots] . »(V, 191)$. Si Benjy, comme le vent, ne s'épuise

\footnotetext{
${ }^{30}$ Plusieurs scènes de La Route des Flandres montreront d'ailleurs d'une façon plus définitive encore la vacuité du cri $(R F, 232)$.

${ }^{31}$ Le Bruit et la Fureur, dernière page.
} 
pas de la brutalité qui le traverse, Montès, Quentin et Muichkine en revanche sont paralysés par cette violence. Les hébétudes de l'idiot s'apparentant à une rigidité cadavérique. Montés voit dans son état " comme une sorte de mort»(136), et affiche "cette expression ahurie, stupéfaite comme par la brusque révélation de la mort» $(R F$, 252) évoquée dans La Route des Flandres: le visage de l'idiot, c'est celui du soldat fauché par un obus.

Les visages d'idiots reparaîtront donc significativement dans La Route des Flandres avec les soldats, les déracinés par excellence, condamnés à une errance, à un bruit et une fureur absurdes, et tout cela hors du temps, comme pour les cavaliers de l'Apocalypse déjà évoqués dans L'Ldiot de Dostoïevski. On dénombre de l'avis de Georges et Blum " cet idiot de sous-lieutenant ", " l'autre orgueilleux imbécile " qu'est l'ancêtre Reixach, et son descendant de Reixach, "cet autre idiot de sabreur ». Blum est une " espèce de con » pour Wack, l'idiot suprême tout désigné par son patronyme (wacky) ${ }^{32}$. Georges s'interroge d'ailleurs sur l'expression du visage de de Reixach mort :

Et je me demandais s'il avait alors lui aussi cet air étonné vaguement offusqué le visage d'idiot de Wack quand il avait été arraché de son cheval gisant mort la tête en bas me regardant de ses yeux grands ouverts la bouche grande ouverte sur le revers du talus, mais lui avait toujours eu une tête d'idiot et bien sûr la mort n'avait pas précisément arrangé les choses de ce point de vue [...]. $(R F, 252)$

Avec Le Vent, un des rares romans simoniens qui n'évoque pas la guerre, Simon propose un idiot, idiot « sans raison ». Sa présence " ne signifie rien », non plus que le monde «- saufqu'il est ${ }^{33}$. C'est sans doute ainsi que l'entend déjà Jean-Paul Sartre dans La Nausée lorsqu'il fait dire à Roquentin : " Exister, c'est être là, tout simplement ».

\footnotetext{
${ }^{32}$ L'histoire rapportée par Georges n'étant elle-même qu'une « vulgaire histoire de cul entre une putain et deux imbéciles ».

${ }^{33}$ Selon l'expression d'Alain Robbe-Grillet, Pour un nouveau roman, Paris, Minuit, 1961, p. 18.
} 
L'épigraphe empruntée à L'Église de Céline en exergue à $\mathrm{La} \mathrm{Nau-}$ sée : "C'est un garçon sans importance collective, c'est tout juste un individu. » engage à pousser la réflexion dans le sens d'une comparaison. Être "sans importance collective» revient pour Montès à représenter un danger pour la collectivité, comme les héros sartriens. L'Autodidacte dans le roman de Sartre porte comme Montés « des vêtements râpés ", " a l'air d'un martyr $~_{34}$, a choisi l'ordre alphabétique pour ordonner sa vie, et demeure stupéfait devant les photographies de Roquentin comme Montés devant celles du narrateur. Roquentin fréquente assidûment les bancs de jardins publics et les hôtels miteux, il éprouve la même fascination navrée que Montés pour l'écoulement du temps et le spectacle des joueurs de cartes ${ }^{35}$.

Pour Roquentin aussi l'indépendance des objets coïncide avec l'angoisse de la désincarnation de l'individu face au temps : " mes yeux allaient rapidement des uns aux autres pour les surprendre, et les arrêter au milieu de leur métamorphose $»^{36}$. Cette vision dénonce chez Sartre l'approche d'une crise de nausée. Lorsque Montés ne peut plus digérer le réel et ses trop nombreuses " mutations ", il évoque l'épisode qui l'a conduit au restaurant après la mort de Rose : « [...] une sorte d'anorexie qui le mettait dans l'impossibilité d'assimiler, non la nourriture (il mangea [...] quoiqu'il eût été incapable de dire [...] quelle sorte d'aliments c'était [...]), mais le monde extérieur devenu quelque chose d'informe $[\ldots] »(V, 138-139)$. Montés ira finalement restituer son déjeuner aux lavabos. Cette indigestion de réel, ces crises de nausée, se manifesteront chez d'autres héros simoniens, par exemple dans Le Palace lorsque l'étudiant ne parvient plus à supporter l'absurdité des événements non plus que leur transformation en mots indigestes dans le journal, et se qualifie finalement de « bougre d'idiot » $(P, 516)^{37}$.

\footnotetext{
${ }^{34}$ La Nausée, op. cit., p. 52 et 166.

35 "En somme, ce fameux écoulement du temps, on en parle beaucoup, mais on ne le voit guère ». Cette obsession de l'invisibilité des cycles de la nature reparaîtra à l'exergue de L'Herbe: "Personne ne fait l'Histoire, on ne la voit pas, pas plus qu'on ne voit l'herbe pousser. Pasternak ».

${ }^{36}$ La Nausée, p. 116.

${ }^{37}$ Voir aussi le malaise du narrateur d'Histoire : «Comment ? Peur ? Si j'avais... Je ne sais pas. Peut-être est-ce comme cela que ça s'appelle : une sensation de nausée [...] " (Hist, 177), et des Corps conducteurs, percevant la marée des passants " [...] entourés de cette nauséeuse aura d'irréalité [...], les étincelantes façades de verre et de métal dont les portes vomissent des groupes bariolés [...] " $(\mathrm{CC}, 72)$.
} 
La compacité indigeste du réel et de ses foisonnants éléments, les "vitres invisibles " auxquelles se heurte l'idiot dans Le Vent, seront des motifs récurrents désormais du roman simonien. Le personnage simonien se caractérisera toujours par une sorte de marginalité, de sauvagerie, d'inadaptabilité à la vie en société : c'est Georges dans L'Herbe qui préfère la terre à la compagnie des humains, c'est le narrateur d'Histoire se soustrayant à trois reprises à des invitations, c'est Jean-Marie L.S.M., le conjuré dans Les Géorgiques, c'est S. dans Le Jardin des Plantes, qui s'illustre par son exclusion des actes consensuels du congrès en Union Soviétique, qui ne peut non plus communiquer avec ses maîtresses, qui est désigné enfin à peu près comme le " mouton noir» du colloque de Cerisy.

L'idiot dans Le Vent préfigure les visages d'écrivains des prochains romans. Montès et le narrateur sont les deux faces du romancier : à sa table d'écriture, le narrateur d'Histoire, de La Bataille de Pharsale, de L'Acacia ou du Jardin des Plantes sera assailli comme Montès d'une multitude de perceptions et de souvenirs simultanés qu'il devra ensuite, comme le narrateur du Vent, tenter de réordonner : « [...] assez vite, dit Simon, (et dans Le Vent j'ai expressément formulé cela dans certains passages) j'ai été frappé par l'opposition, l'incompatibilité même, qu'il y a entre la discontinuité du monde perçu et la continuité de l'écriture ${ }^{38}$. D'ailleurs le sous-titre du roman contient deux modalisations : «tentative» envisage l'échec possible, et " restitution » postule l'infidélité à cause d'éléments fragmentaires, voire purement et simplement portés disparus :

[...] tenter de rapporter, de reconstituer ce qui s'est passé, c'est un peu comme si on essayait de recoller les débris dispersés, incomplets, d'un miroir, s'efforçant maladroitement de les réajuster, n'obtenant qu'un résultat incohérent, dérisoire, idiot $[\ldots]^{39} .(V, 4)$

\footnotetext{
38 " Réponses de Claude Simon à quelques questions écrites de Ludovic Janvier », Entretiens, 31, 1972.

${ }^{39}$ Voir aussi : « [...] ce fut cela qu'il vécut, lui : cette incohérence, cette juxtaposition brutale, apparemment absurde, de sensations, de visages, de paroles, d'actes. Comme un récit, des phrases dont la syntaxe, l'agencement ordonné - substantif, verbe, complément - seraient absents. » $(V, 137)$.
} 
Simon ajoute au kaléidoscope proustien (" débris dispersés ») la lacune (" incomplets »). Cette image du miroir brisé sera schématisée à la fin de La Route des Flandres avec l'image de la moitié d'orange. Face au réel, celui qui voit, fût-il comme Montès pourvu d'un œil photographique, demeure comme Maurice « l'œil collé à une serrure en essayant d'imaginer d'après les bruits et les fragments d'images ce que l'étroit champ de vision ne lui permet pas de voir» (100).

Montés affronté à la brutalité du monde et Montés animal, c'est le soldat, c'est le narrateur d'Histoire ou de L'Acacia fuyant dans les fossés comme une bête et faisant l'expérience d'un retour salvateur à la brutalité. Montés aux prises avec la veste indocile, c'est l'écrivain lui-même aux prises avec les mots et leur émergence arbitraire. À ce sujet Madeleine Chapsal dit de Simon qu'« il parle comme il écrit : sans un point, sans une virgule, avec des parenthèses, des reprises - ce que lui-même, modestement, appelle "bégayer" $»^{40}$. L'œuvre idiote, c'est celle qui bégaye et se répète, se reformule, se refictionnalise indéfiniment, c'est le roman simonien dont chaque livre est aux précédents un "complément d'informations $\|^{41}$. L'acuité du regard de Montés, c'est celle de l'écrivain ayant recours mille fois aux transpositions d'arts plastiques dans ses romans. L'appareil de photographie, c'est le narrateur réduit à son seul regard dans Triptyque. L'œuvre idiote, c'est le palimpseste de La Bataille de Pharsale, ce sont les diagonales du Jardin des Plantes qui se lit « dans tous les sens $»^{42}$. Enfin, Montés errant sur les routes et trébuchant sur les mots, c'est l'écrivain lancé sur les " sentiers de la création » et " découvrant à tâtons le monde dans et par l'écriture » $(O A, 1183)$.

Le visage de l'idiot Montés figure donc le visage même de l'écrivain, ou du poète ${ }^{43}$, et il n'y a sans doute pas de hasard si le romancier a choisi Wack, l'idiot de La Route des Flandres, comme Bellérophon moderne. C'est en effet l'idiot qui dompte Pégase, monture mythique symbole de la création poétique, et née du sang de Mé-

\footnotetext{
${ }^{40}$ L'Express, 10 novembre 1960.

${ }^{41}$ Titre envisagé pour L'Acacia.

${ }^{42}$ Quatrième de couverture du roman.

${ }^{43}$ Voir la correspondance de Simon avec Jean Dubuffet aux Éditions de l'Échoppe ; voir aussi Jean-Paul Sartre, L'Idiot de la famille, t.I, p. 31: "Cette double appartenance simultanée de l'âme au monde et du monde à l'âme, Flaubert l'appelle, quand elle fait l'objet d'une expérience concrète et vécue, tout simplement la Poésie»
} 
duse - celle qui, bien avant Montès et son « troisième œil », pétrifiait instantanément ceux qui croisaient son regard. 